Gisele Chagas de Medeiros

\title{
Preditores clínicos do risco de disfagia após intubação orotraqueal prolongada
}

Tese apresentada à Faculdade de Medicina da Universidade de São Paulo para obtenção do título de Doutor em Ciências

Programa de Ciências da Reabilitação

Orientadora: Profa. Dra. Claudia Regina Furquim de Andrade

São Paulo

2015 
Dados Internacionais de Catalogação na Publicação (CIP)

Preparada pela Biblioteca da

Faculdade de Medicina da Universidade de São Paulo

Creprodução autorizada pelo autor

Medeiros, Gisele Chagas de

Preditores clínicos do risco de disfagia após intubação orotraqueal prolongada / Gisele Chagas de Medeiros. -- São Paulo, 2015.

Tese(doutorado)--Faculdade de Medicina da Universidade de São Paulo.

Programa de Ciências da Reabilitação.

Orientadora: Claudia Regina Furquim de Andrade.

Descritores: 1.Deglutição 2.Transtornos da deglutição 3.Intubação intratraqueal 4.Pneumonia aspirativa 5.Unidades de terapia intensiva 6.Fonoaudiologia

USP/FM/DBD-337/15 
Dedico esse trabalho aos meus pais por me amarem incondicionalmente. 


\section{AGRADECIMENTOS}

Agradeço a Deus, por me conduzir ao encontro das oportunidades e pessoas que foram fundamentais ao meu desenvolvimento pessoal e profissional.

À minha orientadora Profa. Dra. Claudia Regina Furquim de Andrade, exemplo de caráter, integridade e respeito à profissão, pela acolhida, confiança, incentivo e por me permitir a usufruir de seus conhecimentos. A seriedade com que conduz sua atividade científica constitui-se em modelo de atitude para ser seguido.

À fonoaudióloga e amiga Dra. Fernanda Chiarion Sassi por toda a contribuição na construção deste trabalho e por compartilhar seus conhecimentos. Agradeço pela disponibilidade, apoio, carinho e por ser um exemplo profissional e pessoal.

À banca de qualificação do doutorado, Profa. Dra. Débora Maria Befi Lopes, Dra. Fernanda Chiarion Sassi e Dr. Lucas Santos Zambom pelas contribuições assertivas para a finalização deste trabalho. 
À fonoaudióloga e amiga Ms. Dicarla Motta Magnani pelo apoio, paciência e carinho durante a execução desta pesquisa e por ser uma grande parceira de trabalho.

À fonoaudióloga e amiga Dra. Fabíola Staróbole Juste pelo grande exemplo profissional e pessoal, pelas palavras carinhosas e incentivadoras.

À fonoaudióloga e amiga Dra. Danielle Pedroni Moraes pelo companheirismo durante todos esses anos e por todos os trabalhos construídos juntas, o que foi essencial para a construção desta pesquisa.

À fonoaudióloga Dra. Laura Davison Mangilli pelas imprescindíveis colaborações no desenvolvimento do mestrado e por todo o apoio durante esses anos de convivência.

À fonoaudióloga e amiga Ms. Aline Rodrigues Padovani pelo apoio nos meus primeiros anos de atuação na Fonoaudiologia e por ser sido uma grande incentivadora no ingresso à pós-graduação.

À fonoaudióloga e amiga Dra. Silmara Rondon por dividir comigo as angústias e alegrias desse longo caminho. 
Às fonoaudiólogas Juliana Ferrucci, Amanda Pagliotto e Tharsila Moreira pela dedicação e apoio constante na construção de uma atuação fonoaudiológica responsável e de qualidade na Internação do ICHC-FMUSP.

Às fonoaudiólogas atuantes na Internação do ICHC-FMSUP pela dedicação e competência na assistência fonoaudiológica.

Aos alunos e ex-alunos da Divisão de Fonoaudiologia do ICHC-FMUSP por serem os grandes incentivadores nas atualizações científicas constantes. Em especial aos alunos Daniel Sampaio, Renata Moreira e Renata Medeiros por colaborarem diretamente na coleta de dados deste trabalho.

À todos os fonoaudiólogos da Divisão de Fonoaudiologia do HCFMUSP pelos conhecimentos compartilhados nas diversas áreas da Fonoaudiologia.

Aos secretários Francisco Góes, Juliana Santos e Gisele Lúcia pela disponibilidade e ajuda durante a execução deste trabalho. Ao Francisco em especial por ter auxiliado diariamente na organização da coleta de dados.

Ao Aristides Correia pela atenção e disponibilidade durante a análise estatística desta pesquisa.

Aos pacientes que são o maior sentido da realização deste trabalho. 
Ao Dr. Marcelo Park e Dra. Amanda Maria Ribas Rosa de Oliveira pela atenção e auxílio no desenvolvimento desta pesquisa.

À equipe multiprofissional do ICHC-FMSUP pela troca de conhecimentos e por valorizarem a assistência fonoaudiológica nas Unidades de Terapia Intensiva.

Às fonoaudiólogas e amigas Flavia Aires e Camila Bernardes por compartilharem comigo o interesse na atuação fonoaudiológica em Disfagia e pelo companheirismo.

Aos meus pais pelo amor incondicional, carinho, paciência, por terem me estimulado a sonhar e o mais importante, terem me ensinado a enfrentar os desafios para alcançar os meus objetivos.

Ao meu irmão Bruno pelo carinho e exemplo de persistência.

À minha tia Leila pelo companheirismo, amor e força em todas as etapas do meu crescimento. Obrigada por estar sempre ao meu lado, em todas as conquistas e principalmente durante todo o meu percurso profissional.

Ao meu tio João pelo apoio no início da minha carreira profissional. 
Aos meus familiares - tios, avós, primos - por me fazer sentir amada e por sempre estarem de braços abertos me esperando. Obrigada por serem meu porto seguro.

À minha amiga e irmã Tatiana por ser tão companheira. Obrigada pelos anos de convívio, pela paciência, por estar comigo diariamente nos momentos alegres, tristes, confusos, mas sempre felizes.

Ao meu amigo Cassio pela amizade e pelo apoio durante todo esse processo.

Às minhas amigas Nicolle, Lívia, Juliana, Camila e Roberta por toda a amizade ao longo desses 21 anos e por todos os momentos juntas que com certeza foram importantes para o meu crescimento pessoal e me ajudaram a sempre buscar novas possibilidades. Obrigada por manterem a amizade e o amor mesmo com a ausência.

À todos os meus amigos que conquistei em São Paulo. Obrigada pela acolhida desde o começo, pelo carinho e por todos os momentos que fizeram com que eu me sentisse especial.

Ao Marco pelo carinho e por fazer meus dias mais leves no final deste processo. 


\section{EPÍGRAFE}

A mente que se abre a uma nova ideia jamais voltará ao seu tamanho original (Albert Einstein) 


\section{NORMALIZAÇÃO ADOTADA}

Esta dissertação está de acordo com as seguintes normas, em vigor no momento desta publicação:

Referências: adaptado de International Committee of Medical Journals Editors (Vancouver)

Universidade de São Paulo. Faculdade de Medicina. Divisão de Biblioteca e Documentação. Guia de apresentação de dissertações, teses e monografias. Elaborado por Anneliese Carneiro da Cunha, Maria Julia de A. L. Freddi, Maria F. Crestana, Marinalva de Souza Aragão, Suely Campos Cardoso, Valéria Vilhena. 3a ed. São Paulo: Divisão de Biblioteca e Documentação; 2011.

Abreviaturas dos títulos dos periódicos de acordo com List of Journals Indexed in Index Medicus. 


\section{SUMÁRIO}

Lista de Siglas e Abreviaturas

Lista de Quadros

Lista de Tabelas

Lista de Gráficos

Resumo

Summary

1 APRESENTAÇÃO

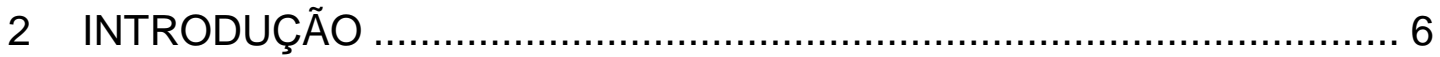

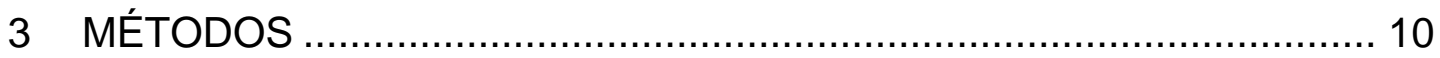

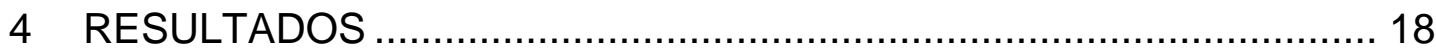

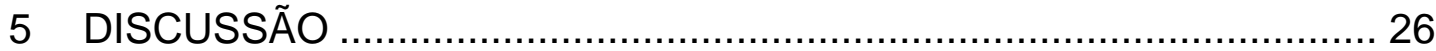

6 CONCLUSÃO

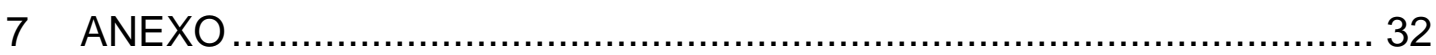

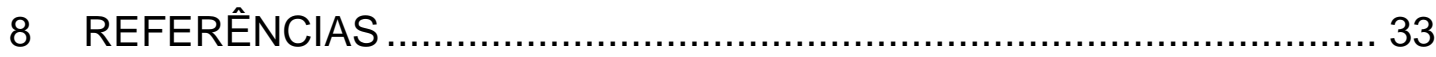




\section{LISTA DE SIGLAS E ABREVIATURAS}

$\checkmark$ IOTP - Intubação orotraquel prolongada

$\checkmark$ UTIs - Unidades de Terapia Intensiva

$\checkmark$ ICHC-FMUSP - Instituto Central do Hospital das Clínicas Faculdade de Medicina da Universidade de São Paulo

$\checkmark$ InCor-FMUSP - Instituto do Coração do Hospital das Clínicas Faculdade de Medicina da Universidade de São Paulo

$\checkmark$ HCFMUSP - Hospital das Clínicas Faculdade de Medicina da Universidade de São Paulo

$\checkmark$ SOFA - Sequential Organ Failure Assessment

$\checkmark$ APACHE II - Acute Physiology and Chronic Health Evaluation II

$\checkmark$ CAPPesq-Comitê de Ética para Análise de Projetos de Pesquisa

$\checkmark$ ASHA NOMS - American Speech-Language-Hearing Association National Outcome Measurement System

$\checkmark$ PARD - Protocolo Fonoaudiológico de Avaliação do Risco da Disfagia

$\checkmark$ DP - Desvio padrão

$\checkmark$ ASHA $1=$ níveis 1 e 2 no American Speech-Language-Hearing Association National Outcome Measurement System

$\checkmark$ ASHA $2=$ níveis 3, 4 e 5 no American Speech-Language-Hearing Association National Outcome Measurement System

$\checkmark$ ASHA $3=$ níveis 6 e 7 no American Speech-Language-Hearing Association National Outcome Measurement System 


\section{LISTA DE QUADROS}

Quadro 1. Definição das variáveis do Protocolo Fonoaudiológico de Avaliação do Risco da Disfagia (PARD) para o teste de deglutição de água $(5 \mathrm{~mL})$.............................................. 12

Quadro 2. Nível funcional da deglutição segundo a American SpeechLanguage-Hearing Association National Outcome Measurement System (ASHA NOMS) ............................... 14

Quadro 3. Sistema de pontuação na Sequential Organ Failure Assessment (SOFA) ..................................................... 16 


\section{LISTA DE TABELAS}

Tabela 1. Resultados na escala do nível de deglutição ASHA NOMS.. 19

Tabela 2. Idade e tempo de intubação orotraqueal dos pacientes 19

Tabela 3. Comparação entre os níveis de deglutição e os preditores do risco de aspiração broncopulmonar

Tabela 4. Múltiplas comparações entre os níveis de deglutição e a gravidade do paciente 22

Tabela 5. Comparação entre gravidade do sistema respiratório, cardiovascular e sistema nervoso central e os níveis de deglutição 24 


\section{LISTA DE GRÁFICOS}

Gráfico 1. Comparação entre os níveis de deglutição e a gravidade do paciente 22 


\section{RESUMO}

Medeiros GC. Preditores clínicos do risco de disfagia após intubação orotraqueal prolongada [tese]. São Paulo: Faculdade de Medicina, Universidade de São Paulo; 2015. 38p.

INTRODUÇÃO: A deglutição é um processo complexo que requer a coordenação precisa de mais de 25 músculos, seis pares de nervos cranianos e os lobos frontais. O comprometimento neste processo, denominado de disfagia, pode aumentar a taxa de morbidade dos pacientes e também o risco para a aspiração, retardando a administração de uma nutrição adequada por via oral. A intubação orotraqueal prolongada, definida na literatura como período superior a 48 horas de intubação, poderá causar alterações na deglutição e ocasionar a disfagia após a extubação. OBJETIVO: correlacionar a gravidade de pacientes críticos não neurológicos com preditores clínicos do risco de broncoaspiração. MÉTODOS: Participaram do estudo adultos, com histórico de intubação orotraqueal prolongada ( $>48$ horas), submetidos à avaliação da deglutição em beira de leito nas primeiras 48 horas após a extubação. A coleta de dados envolveu: avaliação fonoaudiológica clínica do risco de aspiração broncopulmonar; determinação do nível funcional da deglutição (American Speech-Language-Hearing Association National Outcome Measurement System); determinação da gravidade do paciente (Sequential Organ Failure Assessment). RESULTADOS: a amostra do estudo foi composta por 150 pacientes. Para fins da análise estatística, os pacientes foram agrupados da seguinte forma: ASHA 1 (sem possibilidade de alimentação por via oral); ASHA 2 (sem possibilidade de alimentação por via oral); ASHA 3 (deglutição funcional). As análises estatísticas indicaram os seguintes resultados significantes: indivíduos no grupo ASHA 3 eram mais jovens $(46,4 \pm 18,30)$, permaneceram entubados por menos tempo $(4,9 \pm 2,7$ dias) e apresentaram menor gravidade de quadro clínico geral (Sequential Organ Failure Assessment 2,00-5,00). Os sinais clínicos preditores de broncoaspiração que mais diferenciaram os grupos foi a presença de ausculta cervical alterada e presença de tosse após a deglutição, sendo que o grupo ASHA 3 não apresentou esses sinais. CONCLUSÃO: Pacientes críticos, submetidos à IOTP, idade $\geq 55$ anos, com período de intubação $\geq 6$ dias, gravidade de quadro clínico geral $\geq 5$ na Sequential Organ Failure Assessment, pontuação na Escala de Coma de Glasgow $\leq 14$, e com ausculta cervical alterada e tosse após a deglutição, devem ser priorizados para a avaliação fonoaudiológica completa.

Descritores: 1.Deglutição 2.Transtornos da Deglutição 3.Intubação intratraqueal 4.Pneumonia aspirativa 5.Unidades de terapia intensiva 6.Fonoaudiologia 


\section{SUMARY}

Medeiros GC. Clinical predictors of dysphagia risk after prolonged orotracheal intubation [thesis]. São Paulo: "Faculdade de Medicina, Universidade de São Paulo"; 2015. 38p.

INTRODUCTION: Swallowing is a complex process, that require the precise timing and coordination of more than 25 muscles, six cranial nerves and frontal lobes. Compromise of this process, or dysphagia, can result in profund morbidity, increasing the changes of aspiration and delaying the admistration of proper oral nutrition. It is know that an orotracheal tube might disturb these intricately choreographed events and cause post-extubation dysphagia. Prolonged intubation, typically defined as longer than 48 hours in the literature, is thought to contribute to swallowing dysfunction. OBJECTIVES: to correlate the severity of non-neurologic critical patients with clinical predictors for the risk of bronchoaspiration. METHODS: Participants of this study were adults, submitted to prolonged orotracheal intubation ( $>48$ hours) and to a swallowing bedside evaluation during the first 48 hours after extubation. Data gathering involved: clinical assessment of the risk for bronchoaspiration performed by a speech-language pathologist; assessment of the funcitional level of swallowing (American Speech-Language-Hearing Association National Outcome Measurement System); assessment of the patient's health status (Sequential Organ Failure Assessment). RESULTS: the study sampel was composed by 150 patients. For the analyses purposes, patients were grouped as follows: ASHA 1 (individual is not able to swallow safely and alternative feeding is required); ASHA 2 (alternative feeding is required, but individual may receive some nutrition and hydration by mouth); ASHA 3 (swallowing is functional). Statistical analyses indicated that: ASHA 3 patients were younger $(46,4 \pm 18,30$ years), remained intubated for fewer days $(4,9 \pm 2,7)$ and presented a less severe overall health status (Sequential Organ Failure Assessment 2,00-5,00). The clinical predictor signs for bronchoaspiration that best characterized the groups were altered cervical auscultation and presence of cough after swallowing. ASHA 3 patients did not present these signs. CONCLUSION: Critical patients, submitted do POTI, with ages $\geq 55$ years, intubation period $\geq 6$ dias, overall health status $\geq 5$ Sequential Organ Failure Assessment score, Coma Glasgow Scale $\leq 14$, and presenting altered cervical auscultation and cough during swallowing, should be prioritized for a complete swallowing assessment.

Descriptors: 1.Deglutition 2.Deglutition Disorders 3.Orotracheal Intubation 4.Pneumonia/Aspiration 5.Intensive Care Units 6.Speech, language and hearing 


\section{APRESENTAÇÃO}

A temática desta tese segue a proposta, iniciada na dissertação do mestrado, da importância de uma fundamentação metodológica para a avaliação da deglutição em pacientes submetidos à intubação orotraqueal prolongada (IOTP) nas Unidades de Terapia Intensiva (UTIs).

$\mathrm{Na}$ área hospitalar, o fonoaudiólogo, integrando a equipe multiprofissional, atua principalmente no gerenciamento dos distúrbios da deglutição - a fim de propiciar o retorno seguro da alimentação por via oral. A disfagia tem alta incidência e prevalência e contribui para o aumento dos índices de mortalidade, morbidade, econômico-financeiro e principalmente, para redução da qualidade de vida dos pacientes e cuidadores. Desta forma, o estabelecimento de diagnósticos adequados, prognósticos e tratamentos são fundamentais.

A literatura atual descreve diferentes instrumentos para avaliação da deglutição, incluindo os testes de triagem. Estes testes visam identificar indivíduos com risco para disfagia, instrumento de grande importância para detectar a alteração da deglutição nos pacientes que apresentam maior risco de broncoaspiração, entre eles, os pacientes submetidos à IOTP.

O melhor conhecimento dos protocolos de avaliação visa padronizar as ações fonoaudiológicas e garantir a qualidade dos serviços oferecidos, 
aplicando assim o conceito da Fonoaudiologia Baseada em Evidências. Essa perspectiva, que tem feito parte do meu percurso profissional desde a minha graduação, é a base que impulsionará a Fonoaudiologia tanto nas pesquisas clínicas, quanto na assistência nas diversas áreas da Fonoaudiologia. Acredito que o crescimento e a constituição de uma base sólida da Fonoaudiologia - principalmente em instituições dinâmicas e multiprofissionais como a área hospitalar - ocorrerá com a aquisição do conhecimento de técnicas padronizadas e validadas de diagnóstico.

No ano de 2006 concluí minha graduação no Curso de Fonoaudiologia do Centro de Ensino Superior de Juiz de Fora. Nos quatro anos em que desenvolvi minhas atividades discentes me interessei pela área de Disfagia, por ser um campo de ação de grande importância no ambiente hospitalar.

No fim da graduação o interesse pela disfagia fez com que buscasse embasamento científico e prática clínica a fim de ter segurança para exercer esta atividade profissional. Após um período de busca por esta especialização me inscrevi para o processo de seleção do Aprimoramento Profissional em Fonoaudiologia Hospitalar e Funções Orofaciais do Hospital das Clínicas da Faculdade de Medicina da Universidade de São Paulo, coordenado pela Professora Doutora Claudia Regina Furquim de Andrade. Durante o aprimoramento (2006-2007), realizado com bolsa da Fundação do Desenvolvimento Administrativo, pude desenvolver as habilidades e conhecimentos necessários para a prática profissional. Os estágios foram 
realizados no Instituto Central do Hospital das Clínicas da Faculdade de Medicina da Universidade de São Paulo (ICHC-FMUSP) nos vários serviços da Divisão de Fonoaudiologia. Também estagiei nas unidades de internação do Instituto do Coração do Hospital das Clínicas da Faculdade de Medicina da Universidade de São Paulo (InCor-FMUSP). Durante esse período, meu aprendizado foi de grande importância para minha atividade profissional, pois adquiri experiência clínica e grande conhecimento teórico.

Os anos que se seguiram foram importantes e determinantes tanto para o fortalecimento do trabalho junto à equipe da Profa. Dra. Claudia Regina Furquim de Andrade, quanto para meu início da atuação com os alunos da pós-graduação nas diversas UTIs do ICHC-FMUSP. Também foi importante para minha inserção junto às equipes e comissões multiprofissionais do HCFMUSP.

Após o Aprimoramento Profissional iniciei minhas atividades profissionais atuando em atendimentos domiciliares e em hospital particular. Também permaneci no ICHC-FMUSP a convite da Profa. Dra. Claudia Regina Furquim de Andrade, realizando atividades de monitoria do Aprimoramento Profissional em Fonoaudiologia Hospitalar e Funções Orofaciais do Hospital das Clínicas da Faculdade de Medicina da Universidade de São Paulo (HCFMUSP) e estudando temáticas que poderiam gerar um projeto de pesquisa para o mestrado. 
No ano de 2010, fui contratada pela Divisão de Fonoaudiologia do ICHC-FMUSP com o cargo de Fonoaudióloga para desempenhar atividades assistenciais nas UTIs, Unidades de Internação e Pronto Socorro do ICHCFMUSP. Após dois anos de atuação fui nomeada pela Divisão de Fonoaudiologia do ICHC-FMUSP para o cargo de Assistente Técnico II. Este cargo tem o objetivo de gerenciar as atividades desempenhadas pelos fonoaudiólogos junto às UTIs, Unidades de Internação e Pronto Socorro ICHC-FMUSP.

Neste período, ingressei no mestrado, sob orientação da Profa. Claudia Regina Furquim de Andrade e estudei a caracterização dos aspectos funcionais da deglutição de pacientes submetidos à IOTP e investiguei os sinais clínicos preditores do risco de broncoaspiração neste grupo.

Este estudo representou a maior amostra de pacientes brasileiros submetida à IOTPp, que foi avaliada para possível identificação de sinais de broncoaspiração. Até o presente momento, este é um dos poucos estudos que investiga possíveis preditores do risco de broncoaspiração, com base nos sintomas clínicos, em pacientes de UTI, tendo sido publicado como artigo científico em periódico internacional no ano de 2014, no periódico Clinical Science, com o título "Clinical dysphagia risk predictors after prolonged orotracheal intubation". 
Em 2013 ingressei no doutorado, almejando ampliar o conhecimento na área. Desta forma, o projeto teve por objetivo contemplar, além da avaliação da deglutição, o estado de saúde geral dos pacientes e o grau de gravidade do paciente crítico, investigando a relação com as alterações de deglutição. 


\section{INTRODUÇÃO}

A deglutição é um processo complexo que requer a coordenação precisa de mais de 25 músculos ${ }^{1,2}$, envolvendo a contração de múltiplos músculos orofacias, faríngeos, laríngeos, respiratórios e esofágicos ${ }^{3,4}$; seis pares de nervos cranianos e os lobos frontais ${ }^{5,6}$. O comprometimento neste processo, denominado de disfagia, pode aumentar a taxa de morbidade dos pacientes e também o risco para a aspiração, retardando a administração de uma nutrição adequada por via oral ${ }^{1,2}$. Para prevenir a aspiração, uma série de eventos são desencadeados a partir do momento em que o bolo alimentar atinge a parte posterior da cavidade oral, estimulando os neuroceptores que inibem a ação dos músculos envolvidos na respiração, geralmente durante a expiração ${ }^{3,7}$. Em decorrência desta complexa coordenação dos eventos envolvidos na deglutição, é notório que a utilização do tubo orotraqueal possa causar alterações nesse processo, levando à disfagia após a extubação³.

A disfagia pós IOTP - acima de 48 horas ${ }^{5,8-11}$ - é definida como a inabilidade em transferir eficientemente $\mathrm{o}$ alimento da boca ao estômago ${ }^{12}$. Esse tipo de disfagia tem uma incidência que varia na literatura entre 44 a 87\%, 10,11,13-16 dos casos pós extubação, podendo elevar a morbidade e mortalidade ${ }^{17}$. Baseado em dados levantados nos hospitais dos Estados Unidos da América, o custo anual com pacientes disfágicos ultrapassa 500 milhões de dólares ${ }^{12}$. 
De acordo com a estatística americana, aproximadamente 220 mil indivíduos por ano superam quadros críticos associados às insuficiências respiratórias agudas e, portanto, fizeram uso de ventilação mecânica. Desses pacientes, 44 mil irão evoluir com disfagia ${ }^{12}$. Recentemente, uma das preocupações tem sido o estudo das disfagias e seu impacto no sistema de saúde ${ }^{18}$.

No Brasil, essas estimativas específicas não existem, mas sabe-se que para países em desenvolvimento, os cuidados médicos e multidisciplinares intensivos, essenciais para pacientes críticos, pressionam, ainda mais, o planejamento orçamentário que, por si só, já é deficitário ${ }^{19}$. A disfagia pós IOTP retarda o início do retorno para a alimentação oral, aumenta o risco para doenças pulmonares e atrasa a alta hospitalar 20,21,22.

Com base neste cenário, a identificação precoce de preditores que apontem os pacientes com risco de aspiração é extremamente relevante para a priorização dos atendimentos, estabelecimento de condutas adequadas e o retorno mais breve e seguro para a alimentação oral ${ }^{18}$. Vários testes estão disponíveis para o diagnóstico da disfagia pós IOTP, incluindo a avaliação da deglutição em beira de leito, realizada pelo fonoaudiólogo, além dos exames de imagem (videofluoroscopia e videoendoscopia) ${ }^{23}$. Contudo, cabe ressaltar que o uso de tais exames não é a realidade da maioria dos centros de atendimento, além de haver uma limitação quanto às suas indicações para o paciente crítico ${ }^{19}$. 
Estudo recente 23 investigou clinicamente os preditores de risco da disfagia após IOTP, com base nos resultados da avaliação clínica da deglutição em beira leito. Os autores concluem que pacientes que apresentam escape extra oral, deglutições múltiplas, ausculta cervical alterada, engasgos, qualidade vocal alterada e tosse após a deglutição devem ser avaliados prontamente antes de iniciar a reintrodução da alimentação por via oral. Outros estudos que utilizaram avaliações de beira de leito apontam os seguintes sinais como indicativos de risco para aspiração: qualidade vocal após a deglutição $24-26$ e presença de tosse após a deglutição²6,27.

Sabe-se que a gravidade da disfagia após a IOTP está relacionada com o tempo de duração de ventilação mecânica (>48 horas); com escores da Escala de Coma de Glasgow $\leq 14$ ou a idade $>55$ anos ${ }^{5,8-11}$. Porém, há estudos que procuraram verificar a associação da disfagia com outros fatores de risco, como as medidas de gravidade do estado de saúde do paciente. Nesses estudos, os autores incluíram o resultado das escalas que determinam a gravidade dos pacientes no momento da internação - ex. Sequential Organ Failure Assessment (SOFA) e Acute Physiology and Chronic Health Evaluation II (APACHE II) ${ }^{28-30}$.

Dois estudos atuais utilizaram os escores da SOFA, obtidos na admissão dos pacientes na UTI, para investigar suas correlações com a gravidade da disfagia ${ }^{28,29}$. Foi observado, em ambos os estudos, que a gravidade do paciente no momento da internação não apresentou relação 
com a gravidade da disfagia (determinada por avaliação em beira leito). Os pacientes com disfagia leve ou deglutição normal apresentaram valores similares na SOFA quando comparados aos pacientes com disfagia grave. Cabe ressaltar que, nesses estudos, os autores excluíram o dado referente ao sistema nervoso central (Escala de Coma de Glasgow).

Outro estudo ${ }^{30}$, cujo objetivo foi avaliar os fatores demográficos (idade, gênero e raça) e clínicos associados à disfagia pós IOTP em pacientes com alterações pulmonares, analisou os resultados das escalas de gravidade do paciente, SOFA e APACHE II, como possíveis fatores relacionados à gravidade da disfagia após a extubação. Foi observado na análise de regressão linear que quatro variáveis apresentaram significância: duração da intubação; comorbidades gastrointestinais altas; escore da SOFA (indicativo de paciente grave) e valores baixos relacionados com o índice de massa corpórea.

Assim, o objetivo do presente estudo foi correlacionar a gravidade de pacientes críticos não neurológicos (SOFA) com preditores clínicos do risco de broncoaspiração (avaliação fonoaudiológica de beira de leito da deglutição). A identificação das possíveis correlações beneficiará a identificação dos pacientes de alto risco para a broncoaspiração, auxiliando na priorização dos pacientes que necessitam de avaliação fonoaudiológica completa para liberação segura da alimentação por via oral. 


\section{MÉTODOS}

Foi realizado um estudo transversal observacional. $O$ projeto foi aprovado pelo Comitê de Ética para Análise de Projetos de Pesquisa do Hospital das Clínicas da Faculdade de Medicina da Universidade de São Paulo (HCFMUSP CAPPesq Processo 311784 - Anexo 1).

Participaram do estudo pacientes submetidos à avaliação da deglutição em beira de leito, por solicitação médica, atendidos pela Divisão de Fonoaudiologia do ICHC-FMUSP, entre janeiro de 2013 e janeiro de 2015.

Os critérios de inclusão adotados foram: a). apresentar histórico de IOTP (acima de 48 horas) ${ }^{5,8-11}$; b) apresentar idade acima de 18 anos; c) não estar traqueostomizado ou apresentar histórico prévio de traqueostomia; d) não apresentar doenças neurológicas ou neurodegenerativas; e) não apresentar histórico de disfagia esofágica; f) não apresentar câncer de cabeça e pescoço ou histórico de procedimentos cirúrgicos nessas regiões; g) ter sido submetido à avaliação da deglutição em beira de leito nas primeiras 48 horas após a extubação.

As etapas de coleta de dados envolveram a avaliação fonoaudiológica clínica do risco de aspiração broncopulmonar ${ }^{19,23}$, determinação do nível funcional da deglutição segundo a American Speech-Language-Hearing 
Association National Outcome Measurement System (ASHA NOMS) ${ }^{31,32}$ e a determinação da gravidade do paciente segundo a Sequential Organ Failure Assessment (SOFA) ${ }^{33,34}$.

\section{Avaliação fonoaudiológica clínica do risco de broncoaspiração}

O risco de broncoaspiração foi determinado com base no Protocolo Fonoaudiológico de Avaliação do Risco da Disfagia ${ }^{19,23}$. O PARD é um protocolo brasileiro indicado para a avaliação precoce do risco para a disfagia em beira de leito. Sua aplicação inclui a oferta de volumes controlados de água e de purê. O resultado final da avaliação sugere se o paciente pode receber volumes maiores de líquidos/alimentos e diferentes consistências alimentares, além de apontar se existe a necessidade de monitoramento para a alimentação segura. O protocolo é dividido em duas seções - teste de deglutição de água e teste de deglutição com purê. Os resultados observados durante a aplicação são registrados como passa ou falha para cada item do protocolo. Como determinado pelos autores do PARD, os pacientes são avaliados durante a deglutição de 1 a $5 \mathrm{ml}$ de água, oferecidos na seringa e 3, 5 e $10 \mathrm{ml}$ de purê de frutas, oferecidos na colher.

Uma pesquisa publicada recentemente investigou os preditores de risco de disfagia após a intubação orotraqueal prolongada ${ }^{23}$, com base nos resultados do PARD na avaliação com água $(5 \mathrm{ml})$. Nesse trabalho, os autores concluem que os preditores de disfagia nessa população foram: escape extra 
oral; deglutições múltiplas; ausculta cervical alterada; qualidade vocal após a deglutição; presença de tosse após a deglutição e presença de engasgos. Dessa forma, estes foram os itens do PARD considerados para análise e correlação com os demais dados da pesquisa.

Os critérios para interpretação desses dados estão descritos no Quadro 1:

Quadro 1. Definição das variáveis do Protocolo Fonoaudiológico de Avaliação do Risco da Disfagia (PARD) para o teste de deglutição de água $(5 \mathrm{~mL})$

\begin{tabular}{|c|c|c|}
\hline & Variáveis & Julgamento Clínico \\
\hline \multirow{6}{*}{$\begin{array}{c}\text { Teste de } \\
\text { deglutição de } \\
\text { água }(5 \mathrm{ml})\end{array}$} & Escape extra oral & $\begin{array}{l}\text { Passa- Água não escapa pelos lábios, gerencia o bolo } \\
\text { adequadamente } \\
\text { Falha - Dificuldade no gerenciamento do bolo, presença } \\
\text { de escorrimento do líquido pelos lábios }\end{array}$ \\
\hline & Deglutições múltiplas & $\begin{array}{l}\text { Passa - Presença de uma única deglutição por bolo } \\
\text { Falha - Presença de mais de uma deglutição por bolo }\end{array}$ \\
\hline & $\begin{array}{l}\text { Ausculta cervical (o } \\
\text { estetoscópio deve ser } \\
\text { posicionado na parte lateral } \\
\text { da junção entre a laringe e } \\
\text { a traquéia, anterior à artéria } \\
\text { carótida) }\end{array}$ & $\begin{array}{l}\text { Passa - Presença de três sons característicos da } \\
\text { deglutição, indicando que o bolo passou pela faringe - } \\
\text { dois cliques seguidos por um som expiratório } \\
\text { Falha - Quando não há presença dos sons ou presença } \\
\text { de outros sons não descritos acima }\end{array}$ \\
\hline & Voz molhada & $\begin{array}{l}\text { Passa - Não apresenta alterações no primeiro minuto } \\
\text { após a deglutição } \\
\text { Falha - A voz apresenta um som borbulhante ("molhada") } \\
\text { no primeiro minuto após a deglutição }\end{array}$ \\
\hline & Tosse & $\begin{array}{l}\text { Passa - Não há presença de tosse no primeiro minuto } \\
\text { após a deglutição } \\
\text { Falha- Presença de tosse (voluntária ou não) seguida ou } \\
\text { não por pigarro durante o primeiro minuto após a } \\
\text { deglutição }\end{array}$ \\
\hline & Engasgo & $\begin{array}{l}\text { Passa - Não há a presença de engasgo após a deglutição } \\
\text { Falha - Prsença de engasgo durante ou após a deglutição }\end{array}$ \\
\hline
\end{tabular}




\section{Nível funcional da deglutição}

Para determinação do nível funcional da deglutição, foi utilizada a escala ASHA NOMS ${ }^{31,32}$. Essa escala é uma ferramenta multidimensional que verifica o nível de supervisão necessária para a alimentação e as consistências alimentares indicadas para cada paciente, atribuindo um único número entre 1 e 7 (Quadro 2). Para este estudo, o nível da ASHA NOMS foi determinado após a aplicação completa do PARD e, quando necessário, avaliação clínica da deglutição (oferta de refeições completas, com consistências variadas e diferentes volumes, observando os mesmos critérios estabelecidos pelo PARD). 
Quadro 2. Nível funcional da deglutição segundo a American Speech-LanguageHearing Association National Outcome Measurement System (ASHA NOMS)

Nível 1 O individuo não é capaz de deglutir nada com segurança por via oral. Toda nutrição e hidratação são recebidas através de via alternativa de alimentação (ex.: sonda nasogástrica, gastrostomia).

Nível 2 O indivíduo não é capaz de deglutir com segurança por via oral para nutrição e hidratação, mas pode ingerir alguma consistência, somente em terapia, com uso máximo e consistente de pistas. Via alternativa de alimentação é necessária.

Nível 3 Via alternativa de alimentação é necessária, uma vez que o indivíduo ingere menos de $50 \%$ da nutrição e hidratação por via oral; e/ou a deglutição é segura com o uso moderado de pistas para uso de estratégias compensatórias; e/ou necessita de restrição máxima da dieta.

Nível 4 A deglutição é segura, mas frequentemente requer uso moderado de pistas para uso de estratégias compensatórias; e/ou o indivíduo tem restrições moderadas da dieta; e/ou ainda necessita de via alternativa de alimentação e/ou suplemento oral.

Nível 5 A deglutição é segura com restrições mínimas da dieta; e/ou ocasionalmente requer pistas mínimas para uso de estratégias compensatórias. Ocasionalmente pode se auto monitorar. Toda nutrição e hidratação são recebidas por via oral durante a refeição.

Nível 6 A deglutição é segura e o indivíduo come e bebe independentemente. Raramente necessita de pistas mínimas para uso de estratégias compensatórias. Frequentemente se auto monitora quando ocorrem dificuldades. Pode ser necessário evitar alguns itens específicos de alimentos (ex.: pipoca e amendoim); tempo adicional para alimentação pode ser necessário (devido à disfagia).

Nível 7 A habilidade do indivíduo em se alimentar independentemente não é limitada pela função de deglutição. A deglutição é segura e eficiente para todas as consistências. Estratégias compensatórias são utilizadas efetivamente quando necessárias. 


\section{Gravidade do paciente}

Para a determinação da gravidade do paciente no momento da avaliação clínica fonoaudiológica foi utilizada a Sequential Organ Failure Assessment (SOFA) ${ }^{33,34}$, registrada em prontuário médico com base em exames laboratoriais e clínicos.

A SOFA ${ }^{33,34}$ é um instrumento aplicado diariamente em pacientes críticos durante sua permanência na UTI, para determinar, quantitativa e objetivamente, os níveis de disfunção/falência dos órgãos. O escore obtido pela SOFA é utilizado não para determinar o desfecho do paciente, e sim, para descrever as complicações dos pacientes críticos. Os dois maiores objetivos da SOFA são: melhorar a compreensão da disfunção dos órgãos e a relação entre os prejuízos dos vários órgãos, e avaliar os efeitos do tratamento médico adotado.

Para determinar a gravidade do paciente, são atribuídos escores de 0 (normal) a 4 (maior grau de comprometimento) para os diferentes sistemas: respiratório, cardiovascular, hematológico, hepático, sistema nervoso central e renal. Cada sistema recebe um escore separado e a pontuação final é obtida pela soma de todos os escores. A pontuação máxima é 20 , sendo essa indicativa de maior gravidade. Os critérios para atribuição dos pontos estão descritos no Quadro 3. 
Quadro 3. Sistema de pontuação na Sequential Organ Failure Assessment (SOFA)

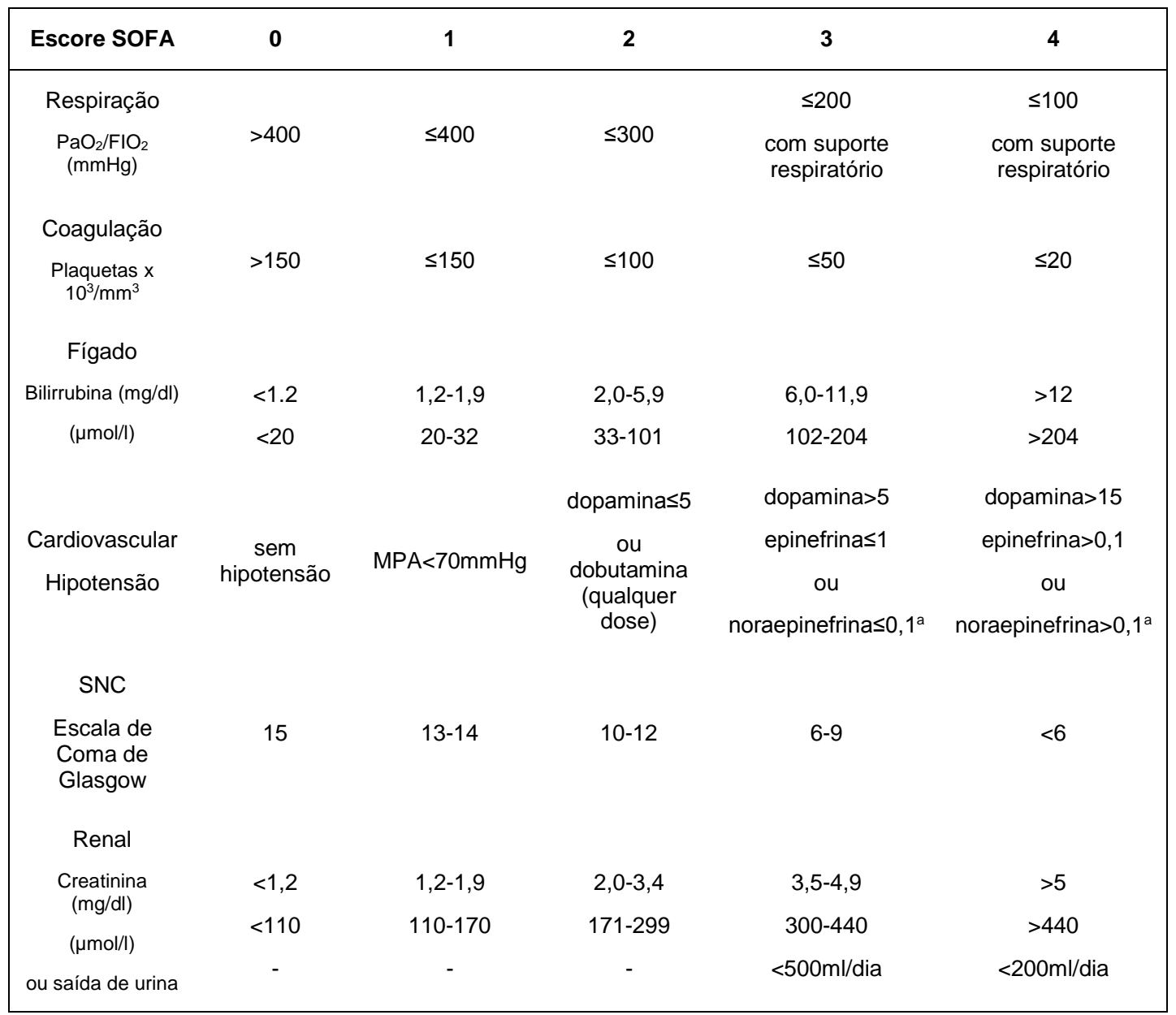

Legenda: SOFA = Sequential Organ Failure Assessment; $\mathrm{PaO}_{2} / \mathrm{FIO}_{2}=$ relação entre a pressão parcial de oxigênio no sangue arterial e a frações inspiradas de oxigênio; $\mathrm{mmHG}=$ milímetros de mercúrio; Plaquetas $\times 10^{3} / \mathrm{mm}^{3}=$ concentração de plaquetas em milímetros cúbicos de sangue; $\mathrm{mg} / \mathrm{dl}=$ miligrams por decilitro; $\mu \mathrm{mol} / \mathrm{l}=$ micromol por litro; MPA = média da pressão arterial; ${ }^{a}$ = agentes adrenérgicos administrados por pelo menos uma hora (doses administradas em micrograma por quilo por minuto); $\mathrm{ml}=$ mililitro.।

\section{Análise dos dados}

Os dados quantitativos com distribuição normal foram apresentados com médias acompanhadas dos respectivos desvios padrão (DP). Os dados quantitativos sem distribuição normal foram expressos através das medianas 
acompanhadas dos respectivos intervalos interquantil IQ (25-75\%). As variáveis categóricas foram expressas através de suas frequências.

Os pressupostos da distribuição normal em cada grupo e a homogeneidade das variâncias entre os grupos foram avaliados, respectivamente, com o teste de Shapiro-Wilk e com o teste de Levene.

Para comparação dos valores da SOFA entre os diferentes níveis funcionais da deglutição foi utilizado o teste de Kruskal Wallis e quando foi necessário realizar comparações múltiplas, foi utilizado o teste de Dunn. As variáveis categóricas foram analisadas com os testes de Qui Quadrado e Exato de Fisher. Neste estudo, o valor de significância estatística adotado foi $5 \%(p \leq 0,05)$. A modelagem estatística e os testes foram realizados com 0 software SPSS versão 21.0. 


\section{RESULTADOS}

Após a aplicação dos critérios de inclusão, a amostra do estudo foi composta por 150 pacientes, 82 homens e 68 mulheres, com média de idade de 53,9 anos $( \pm 18,55)$. Dentre os indivíduos selecionados, as doenças de base registradas em prontuário médico foram: doenças pulmonares, câncer (gastrointestinal e linfoma), politraumas, hepatopatias, doenças renais, transplantes (renal e hepático), doenças cardíacas, doenças reumatológicas, doenças gastroenterológicas e moléstias infecciosas. Os pacientes apresentaram média de tempo de intubação de 6,1 dias $( \pm 3,48)$.

A Tabela 1 apresenta os níveis de deglutição, de acordo com a escala ASHA NOMS, após a avaliação fonoaudiológica em beira de leito. Para fins da análise estatística, os pacientes foram agrupados e renomeados da seguinte forma: níveis 1 e 2 - ASHA 1; níveis 3, 4 e 5 - ASHA 2; níveis 6 e 7 - ASHA 3.

A caracterização dos pacientes quanto a idade e tempo de intubação está descrita na Tabela 2. 
Tabela 1. Resultados na escala do nível de deglutição ASHA NOMS

\begin{tabular}{ccc}
\hline Nível & $\mathbf{n}$ & $\%$ \\
\hline 1 & 3 & 2 \\
2 & 35 & 23 \\
3 & 22 & 15 \\
4 & 27 & 18 \\
5 & 12 & 8 \\
6 & 10 & 7 \\
7 & 41 & 27 \\
\hline
\end{tabular}

Legenda: $\mathrm{n}$ - número de pacientes; \% de pacientes.

Tabela 2. Idade e tempo de intubação orotraqueal dos pacientes

\begin{tabular}{|c|c|c|c|c|}
\hline & ASHA1 & ASHA2 & ASHA3 & p-valor \\
\hline $\begin{array}{c}\text { Idade }( \pm D P) \text { em } \\
\text { anos }\end{array}$ & $62,0 \pm 17,4$ & $55,3 \pm 17,48$ & $46,4 \pm 18,30$ & $<0,001^{*}$ \\
\hline $\begin{array}{c}\text { Tempo de } \\
\text { intubação ( } \pm D P) \\
\text { em dias }\end{array}$ & $7,6 \pm 3,97$ & $6,2 \pm 3,38$ & $4,9 \pm 2,7$ & $<0,001^{*}$ \\
\hline
\end{tabular}

Legenda: DP = desvio padrão; ASHA 1 = níveis 1 e 2 no American Speech-Language-Hearing Association National Outcome Measurement System; ASHA 2 = níveis 3, 4 e 5 no American SpeechLanguage-Hearing Association National Outcome Measurement System; ASHA 3 = níveis 6 e 7 no American Speech-Language-Hearing Association National Outcome Measurement System; * = resultado significante; teste ANOVA

A análise estatística pelo teste de Kruskal Wallis indicou que houve diferença significante para a idade entre o ASHA 1 e o ASHA $3\left(p<0,001^{*}\right)$ e o ASHA 2 e o ASHA $3\left(p=0,026^{*}\right)$, sendo o ASHA3 composto por indivíduos mais jovens. Considerando a média de tempo de IOTP (em dias) para os diferentes grupos, o grupo ASHA 3 foi o que apresentou menor tempo de intubação (diferença significante entre o ASHA1 e o ASHA3; $p=0,001^{*}$ ). 
A Tabela 3 apresenta a comparação entre os diferentes níveis de deglutição e os preditores clínicos do risco para aspiração broncopulmonar.

Tabela 3. Comparação entre os níveis de deglutição e os preditores do risco de aspiração broncopulmonar

\begin{tabular}{|c|c|c|c|c|c|}
\hline Sinais Clínicos & & ASHA 1 (n) & ASHA 2 (n) & ASHA 3 (n) & p-valor \\
\hline \multirow{3}{*}{ Escape extra oral } & Passa & 35 & 57 & 50 & \multirow{3}{*}{0,402} \\
\hline & & & & & \\
\hline & Falha & 3 & 4 & 1 & \\
\hline \multirow{3}{*}{ Deglutições múltiplas } & Passa & 24 & 42 & 44 & \multirow{3}{*}{$0,030^{*}$} \\
\hline & & & & & \\
\hline & Falha & 14 & 19 & 7 & \\
\hline \multirow{3}{*}{ Ausculta cervical } & Passa & 21 & 52 & 51 & \multirow{3}{*}{$<0.001^{*}$} \\
\hline & & & & & \\
\hline & Falha & 17 & 9 & 0 & \\
\hline \multirow{3}{*}{ Voz molhada } & Passa & 31 & 55 & 51 & \multirow{3}{*}{$0.009^{*}$} \\
\hline & & & & & \\
\hline & Falha & 7 & 6 & 0 & \\
\hline \multirow{3}{*}{ Tosse } & Passa & 16 & 37 & 50 & \multirow{3}{*}{$<0.001^{*}$} \\
\hline & & & & & \\
\hline & Falha & 22 & 24 & 1 & \\
\hline \multirow{3}{*}{ Engasgo } & Passa & 31 & 52 & 51 & \multirow{3}{*}{$0,008^{*}$} \\
\hline & & & & & \\
\hline & Falha & 7 & 9 & 0 & \\
\hline
\end{tabular}

Legenda: $\mathrm{n}$ = número de pacientes; ASHA 1 = níveis 1 e 2 no American Speech-Language-Hearing Association National Outcome Measurement System; ASHA 2 = níveis 3, 4 e 5 no American SpeechLanguage-Hearing Association National Outcome Measurement System; ASHA 3 = níveis 6 e 7 no American Speech-Language-Hearing Association National Outcome Measurement System; * = resultado significante; teste Qui-Quadrado.

Os resultados indicaram que o preditor de risco "escape extra oral" não diferenciou os grupos quanto à funcionalidade da deglutição. Os preditores de risco "alteração na ausculta cervical" e a "presença de tosse" após a deglutição são os melhores indicadores de diferenciação dos grupos. Com o objetivo de verificar para quais grupos os preditores de risco de aspiração são 
mais evidentes, foi aplicado o teste Exato de Fisher. Nessa análise foi observado o seguinte resultado: deglutições múltiplas - ASHA 1 versus ASHA $2(p=0,559)$; ASHA 1 versus ASHA $3\left(p=0,011^{*}\right)$; ASHA 2 versus ASHA 3 $\left(p=0,030^{\star}\right)$; ausculta cervical - ASHA 1 versus ASHA $2\left(p=0,001^{*}\right)$; ASHA 1 versus ASHA $3\left(p<0,001^{*}\right)$; ASHA 2 versus ASHA $3\left(p=0,004^{\star}\right)$; voz molhada - ASHA 1 versus ASHA $2(p=0,236)$; ASHA 1 versus ASHA $3\left(p=0,002^{*}\right)$; ASHA 2 versus ASHA $3\left(p=0,031^{*}\right)$; tosse após a deglutição - ASHA 1 versus ASHA 2 ( $p=0,072)$; ASHA 1 versus ASHA 3 ( $\left.p<0,001^{\star}\right)$; ASHA 2 versus ASHA $3\left(p<0,001^{*}\right)$; engasgo - ASHA 1 versus ASHA $2(p=0,630)$; ASHA 1 versus ASHA $3\left(p=0,002^{*}\right)$; ASHA 2 versus ASHA $3\left(p=0,004^{*}\right)$.

O gráfico 1 e a Tabela 4 apresentam os resultados na comparação entre os níveis funcionais da deglutição e a gravidade do paciente (SOFA), no momento da avaliação fonoaudiológica. 
Gráfico 1. Comparação entre os níveis de deglutição e a gravidade do paciente

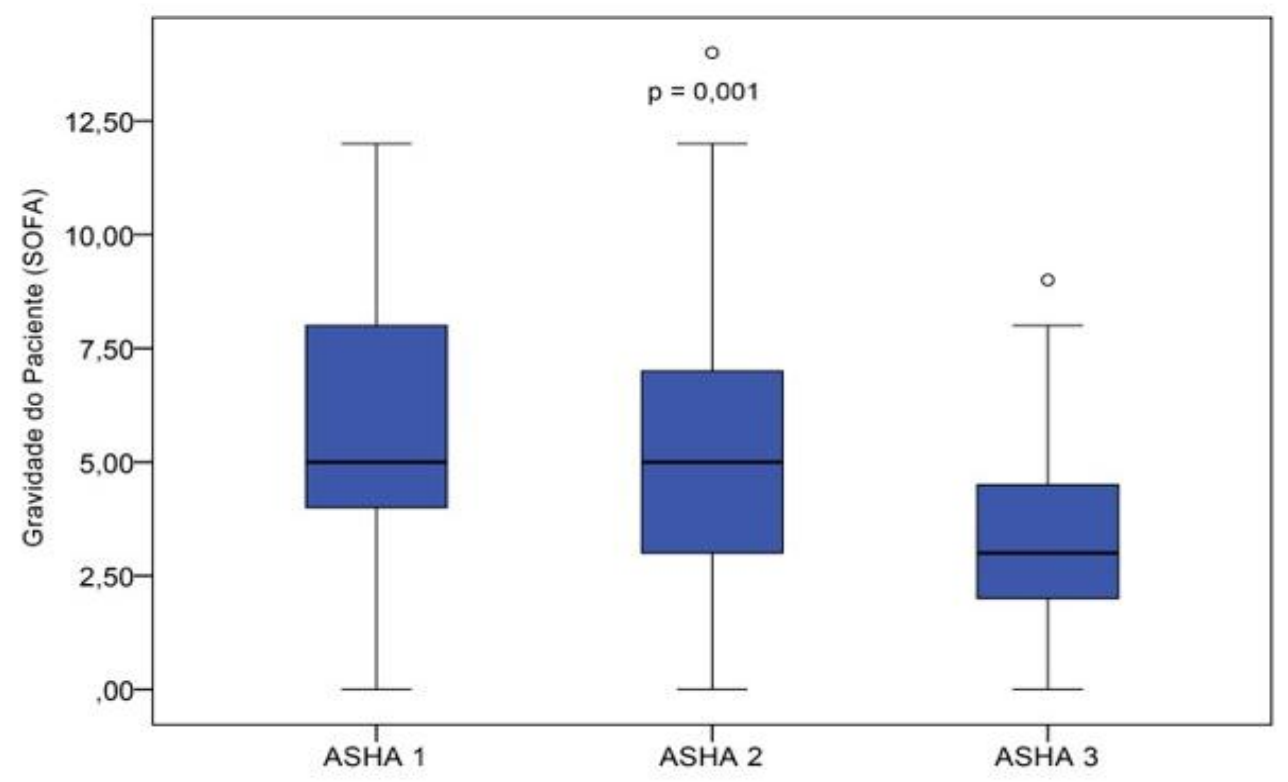

Legenda: ASHA 1 = níveis 1 e 2 no American Speech-Language-Hearing Association National Outcome Measurement System; ASHA 2 = níveis 3, 4 e 5 no American Speech-Language-Hearing Association National Outcome Measurement System; ASHA 3 = níveis 6 e 7 no American Speech-Language-Hearing Association National Outcome Measurement System.

Tabela 4. Múltiplas comparações entre os níveis de deglutição e a gravidade do paciente

\begin{tabular}{cc}
\hline Comparação & p-valor \\
\hline ASHA 1 versus ASHA 2 & $>0.999$ \\
ASHA 1 versus ASHA 3 & $0,003^{*}$ \\
ASHA 2 versus ASHA 3 & $0,005^{*}$ \\
\hline
\end{tabular}

Legenda: ASHA 1 = níveis 1 e 2 no American Speech-Language-Hearing Association National Outcome Measurement System; ASHA 2 = níveis 3, 4 e 5 no American Speech-Language-Hearing Association National Outcome Measurement System; ASHA 3 = níveis 6 e 7 no American Speech-Language-Hearing Association National Outcome Measurement System; ${ }^{*}$ = resultado significante; teste de Dunn. 
Os resultados das duas análises indicam que quando menos funcional a deglutição, maior a gravidade do paciente. Observa-se ainda que o grupo ASHA 1 (sem possibilidade de alimentação por via oral) e o grupo ASHA 2 (com restrição máxima a moderada de consistência de dieta) não se diferenciaram quanto a gravidade dos pacientes na SOFA.

A fim de verificar quais os possíveis indicadores de gravidade do paciente que poderiam ter impacto na deglutição, foi realizada a correlação entre os indicadores de funcionamento dos sistemas respiratório, cardiovascular e de sistema nervoso central da escala SOFA com o nível de funcionalidade da deglutição do paciente no momento da avaliação fonoaudiológica (Tabela 6). 
Tabela 5. Comparação entre gravidade do sistema respiratório, cardiovascular e sistema nervoso central e os níveis de deglutição

\begin{tabular}{|c|c|c|c|c|}
\hline Escala SOFA & ASHA $1(n)$ & ASHA 2 (n) & ASHA $3(n)$ & p-valor \\
\hline \multicolumn{5}{|c|}{ Respiração } \\
\hline 0 & 8 & 13 & 14 & \\
\hline 1 & 14 & 13 & 15 & \\
\hline 2 & 9 & 18 & 7 & 0,436 \\
\hline 3 & 7 & 14 & 13 & \\
\hline 4 & 0 & 3 & 2 & \\
\hline \multicolumn{5}{|c|}{ Cardiovascular } \\
\hline 0 & 28 & 49 & 47 & \\
\hline 1 & 2 & 3 & 1 & \\
\hline 2 & 1 & 1 & 1 & 0,507 \\
\hline 3 & 5 & 7 & 1 & \\
\hline 4 & 2 & 1 & 1 & \\
\hline \multicolumn{5}{|c|}{ SNC (Glasgow) } \\
\hline 0 & 15 & 40 & 43 & \multirow{4}{*}{$0,001^{*}$} \\
\hline 1 & 18 & 18 & 8 & \\
\hline 3 & 4 & 2 & 0 & \\
\hline 4 & 3 & 1 & 0 & \\
\hline
\end{tabular}

Legenda: $\mathrm{n}=$ número de pacientes; ASHA 1 = níveis 1 e 2 no American Speech-Language-Hearing Association National Outcome Measurement System; ASHA 2 = níveis 3, 4 e 5 no American Speech-Language-Hearing Association National Outcome Measurement System; ASHA 3 = níveis 6 e 7 no American Speech-Language-Hearing Association National Outcome Measurement System; SNC = sistema nervoso central; * = resultado significante; teste Qui-Quadrado.

Os resultados indicaram que somente a alteração do sistema nervoso central correlacionou-se com a alteração da funcionalidade da deglutição, indicando que quanto pior a funcionalidade da deglutição, pior a condição do sistema nervoso central. Analisando somente o indicador referente ao sistema 
nervoso central, o teste de Dunn indicou que o grupo com pior funcionalidade da deglutição se diferenciou dos demais: ASHA 1 versus ASHA $2\left(p=0,018^{\star}\right)$; ASHA 1 versus ASHA $3\left(p<0,001^{*}\right)$; ASHA 2 versus ASHA $3(p=0,101)$. 


\section{DISCUSSÃO}

O presente estudo apresenta as características do risco para broncoaspiração do maior grupo de pacientes pós IOTP no Brasil. Até o momento, este é um dos poucos estudos que avalia possíveis preditores de risco para a broncoaspiração em pacientes críticos com base em indicadores clínicos. O estabelecimento de indicadores de priorização de atendimento fonoaudiológico de pacientes pós extubação é primordial para a redução de custos hospitalares, otimização das avaliações fonoaudiológicas em beira de leito e retorno precoce e seguro para a alimentação por via oral.

De maneira geral, os resultados do presente estudo indicaram que 0 grupo ASHA 3 (pacientes que apresentam deglutição segura e eficiente, podendo utilizar estratégias compensatórias com eficiência quando necessário) apresentou idade inferior aos demais (abaixo de 55 anos), apresentou menor tempo de intubação (um terço a menos do que o grupo mais grave), apresentou menor gravidade do quadro clínico geral (mediana equivalente a 3 na SOFA) e nível de consciência normal (Escala de Coma de Glasgow 15). Os sinais clínicos preditores de broncoaspiração que mais diferenciaram os grupos foi a presença de ausculta cervical alterada e presença de tosse após a deglutição, sendo que o grupo ASHA 3 não apresentou esses sinais.

A literatura aponta que o impacto da idade na ocorrência da disfagia pós IOTP é similar em adultos jovens e idosos ${ }^{35-38}$, os grupos parecem se 
diferenciar apenas quanto a resolução da disfagia, sendo que idosos tendem a retornar para a alimentação por via oral mais tardiamente ${ }^{9,27,39}$. Ao contrário da literatura, o presente estudo encontrou que indivíduos com idade superior a 55 anos apresentam maior frequência de sinais preditores de broncoaspiração.

Estudos anteriores, descreveram o impacto do envelhecimento da função da deglutição, indicando que idosos saudáveis apresentam resíduo em valécula (retenção laríngea) e que normalmente, estes indivíduos, não tem consciência dos alimentos que permanecem retidos na faringe ${ }^{40}$. Um desses estudos $^{41}$ descreve que, apesar da limpeza faríngea ser praticamente completa em indivíduos jovens assintomáticos, o mesmo não é observado em idosos saudáveis. Os mecanismos responsáveis pelo desenvolvimento da retenção faríngea do alimento ainda não são bem conhecidos. De acordo com a literatura ${ }^{42-44}$, mudanças fisiológicas podem estar envolvidas no envelhecimento do processo da deglutição - amplitude da contração faríngea reduzida, encurtamento faríngeo, diminuição da propulsão/força de língua e diminuição da força de véu palatino para auxiliar o deslocamento do bolo alimentar. Esses fatores também poderiam justificar a maior frequência da ocorrência de tosse e alteração da ausculta após a deglutição, observada no presente estudo, nos pacientes críticos com idade acima de 55 anos.

A associação entre o tempo da intubação orotraqueal e a gravidade da disfagia é bem respaldada pela literatura ${ }^{5,21,28,45,46}$. Essa associação pode ser explicada pelo impacto da permanência do tubo na cavidade oral, faringe e 
laringe. Sabe-se que os quimiorreceptores e/ou mecanoceptores, localizados nas mucosas da faringe e laringe e envolvidos no reflexo de deglutição, podem sofrer alterações devido a presença do tubo orotraqueal ${ }^{11}$. A inibição das habilidades sensoriais da laringe, identificada pela ausência de tosse ou qualquer outro sinal clínico sugestivo de aspiração, já foi observada em pacientes, durante a ingestão de líquido, tanto no período imediatamente após a extubação quanto no período de até quatro horas pós-extubação ${ }^{47}$.

A maioria das lesões de mucosas causadas pelo tubo orotraqueal apresentam melhora espontânea em até três dias após sua remoção ${ }^{48}$. Entretanto, as alterações de deglutição podem persistir após a retirada do tubo orotraqueal e mesmo após o período de melhora espontânea. A persistência das alterações da deglutição após a extubação pode ser decorrente de uma combinação entre o desuso da musculatura enquanto o tubo permanece na laringe e a perda de propriocepção devido a lesões na mucosa $^{9}$. No presente estudo, os resultados sugerem que indivíduos que permaneceram intubados por mais de seis dias apresentaram maior frequência de sinais preditores do risco de aspiração.

O presente estudo identificou a ausculta cervical alterada como possível preditor do risco da broncospiração. A ausculta cervical é um método não invasivo que tem sido utilizado para complementar a avaliação da deglutição desde os anos $60^{49}$. Os sons associados à deglutição saudável e patológica já foram identificados com o auxílio de acelerômetros e microfones, 
a fim de analisar as características acústicas ${ }^{50}$ e determinar os sons característicos da deglutição ${ }^{50-52}$. Os resultados sobre a precisão do método variam consideravelmente quanto à confiabilidade e validade quando comparados aos exames de imagem ${ }^{53,54}$. Em contrapartida, deve se considerar que a própria interpretação das imagens e resultados da videofluoroscopia também apresentam variações de confiabilidade e reprodutibilidade 55,56 . Independentemente do método adotado para avaliar a deglutição, é consenso que o treinamento específico para aplicação dos mesmos é indispensável.

A análise dos dados indicou que no presente estudo, pacientes críticos submetidos à IOTP, que apresentaram pior nível funcional da deglutição, apresentam pior quadro clínico geral, sendo a alteração do sistema nervoso central o fator com maior impacto nessa diferença. Conforme apresentado na introdução, os resultados dos estudos que correlacionaram a gravidade do quadro clínico do paciente pela SOFA com a gravidade da disfagia não encontraram significância 28,29 . Contudo, o item referente ao comprometimento do sistema neurológico não foi incluído na análise desses mesmos estudos. A literatura já identificou que o nível rebaixado de consciência tem impacto no mecanismo da deglutição, inclusive podendo levar à aspiração9,13,46.

Finalmente, cabe discutir as limitações do estudo aqui apresentado. Em primeiro lugar, a amostra de pacientes utilizada para a composição deste estudo foi oriunda de uma única instituição e, portanto, os resultados podem 
apresentar algum tipo de viés decorrente das abordagens terapêuticas adotadas nos protocolos específicos. Além disso, por se tratar de um hospital terciário, os casos referidos para esta unidade são de alta complexidade e gravidade. Em segundo lugar, o tipo de avaliação proposta para a identificação dos preditores de risco de broncoaspiração foi baseada exclusivamente em protocolo observacional clínico. A verificação real da ocorrência da broncoaspiração, com base nos sinais avaliados, não foi verificada objetivamente com os exames de imagem. Cabe ressaltar que 0 presente estudo não exclui a avaliação fonoaudiológica completa, incluindo os exames de imagem.

O objetivo principal proposto e atingido neste estudo foi fornecer indicadores de priorização dos atendimentos fonoaudiológicos, permitindo identificar os pacientes de maior risco para a aspiração e encaminhá-los para os procedimentos pertinentes, garantindo um retorno mais breve e seguro da alimentação por via oral, a retirada precoce da via alternativa de alimentação, prevenindo assim, a ocorrência de pneumonia aspirativa. 


\section{CONCLUSÃO}

Os resultados do presente estudo sugerem que pacientes críticos, submetidos à intubação orotraqueal prolongada, com idade superior a 55 anos, que permaneceram intubados por mais de seis dias, com gravidade de quadro clínico geral igual ou superior a 5 na SOFA, pontuação na Escala de Coma de Glasgow igual ou menor a 14 e que apresentam ausculta cervical alterada e tosse após a deglutição no teste de água $(5 \mathrm{~mL})$ em beira de leito, devem ser priorizados para a avaliação fonoaudiológica completa e se necessário, encaminhados para a confirmação dos resultados por exame de imagem. 


\section{ANEXO}

\section{Hospital das Clínicas da FMUSP}

Comissdo de Ética para Análise de Projetos de Pesquisa - CAPPesq

\section{PROJETO DE PESQUISA}

ThULO: PREDUTORES CJNICOS DO ROCO DE DSFAMIA APOS INTUBAÇAOO CAOTRAQUEAL PROLIONGADA

Pevalsador Responsivel: Claudia Regina Furquim de

Verste: 1

Andrade

Peveulador Enecutante: Gisele Chagas de Medeires

CAAE: 17340913.900000068

Finalidade Acadintica Doutorado

Instituik: Ae: HCFMUSP

Departamemo: FISIOTERAPLA, FONOAUDIOLOGIA E TERAFA DCUPNONALI

PARECER CONSUBSTANCIADO DO CEP

Registro an-line: 10914

Nümero do Parecer $311.7 \mathrm{n}$

Dena de Relatoria: $19 / 06 / 2013$

Apresentopjo ds Frejete Projmo ben ebsoorads, claro, com objettrss bem definidos.

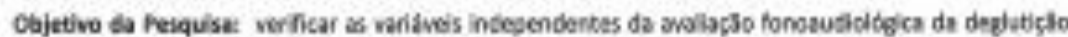

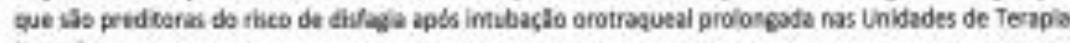
intansive

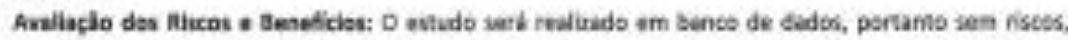

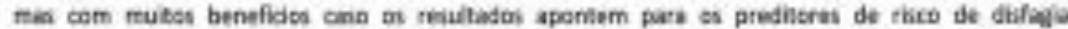
tuscados.

Comentirios e Considerapbes sobre a Pesquiba: Trata-se de pesquisa en banco de datas com finakdade de doutoramento. o estudo esta ben selineaso e deverd traser resaltados ope prepsrdienarbo avanģe do ctontweiminto.

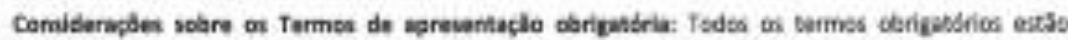

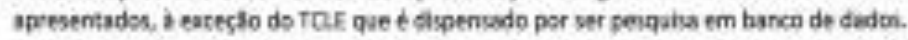

Becomendaptec adin.

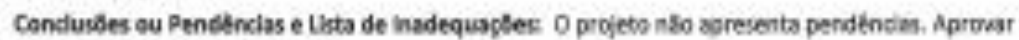

Shuagso do Parecen Aarueado

Necenahe Apredaçlo de conep: Nas

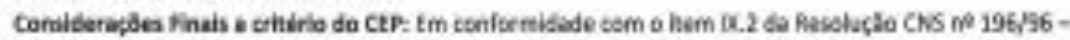

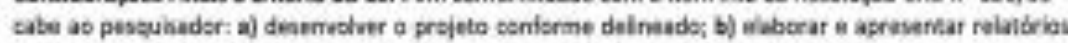

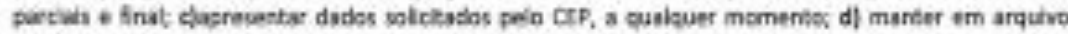

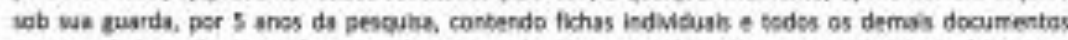

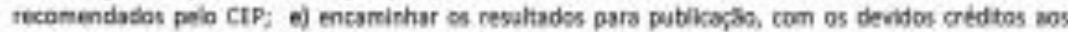

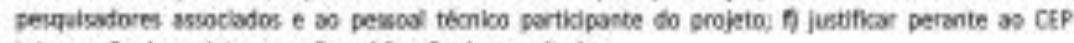
interrusclo do projeto ou a nso publcaclo dos resulados. 


\section{REFERÊNCIAS}

1. Bordon A, Bokhari R, Sperry J, Testa D, Feinstein A, Ghaemmaghami V. Swallowing dysfunction after prolonged intubation: analysis of risk factors in trauma patients. Am J Surg. 2011; 202(6):679-82.

2. Kozlow JH, Berenholz SM, Garret E Epidemiology and impact of aspiration pneumonia in patients undergoing surgery in Maryland, 1999-2000. Crit Care Med. 2003; 31(7):1930-7.

3. Heffner JE. Swallowing complications after endotracheal extubation: moving from "wether" to "how". [editorial] Chest. 2010; 137(3): 509-10.

4. Klahn MS, Perlman Al. Temporal and durational patterns associating respiration and swallowing. Dysphagia 1999; 14(3):131-8.

5. Brown CVR, Hejl K, Mandaville AD, Chaney PE, Stevenson G, Smith C. Swallowing dysfunction after mechanical ventilation in trauma patients. $J$ Crit Care. 2011; 26(1): 108.e9-13.

6. Morgan AS, Mackay LE. Causes and complications associated with swallowing disorders in traumatic brain injury. J Head Trauma Rehabil. 1999; 14: 454-61.

7. Martin-Harris B, Brodsky MB; Price CC, Michel Y, Walters B. Temporal coordination of pharyngeal and laryngeal dynamics with breathing during swallowing: single liquid swallows. J Appl Physiol. 2003; 94(5): 1735-43.

8. Ajemian MS, Nirmul GB, Anderson MT, Zirlen DM, Kwasnik EM Routine fiberoptic endoscopic evaluation of swallowing following prolonged intubation implications for management. Arch. Surg. 2001; 136(4): 434-7.

9. Barquist E, Brown M, Cohn S, Lundy D, Jackowski J. Postextubation fiberoptic endoscopic evaluation of swallowing after prolonged endotracheal intubation: a randomized, prospective trial. Crit Care Med. 2001; 29(9):1710-13. 
10. Leder SB, Cohn SM, Moller BA. Fiberoptic endoscopic documentation of the high incidence of aspiration following extubation in critically ill trauma patients. Dysphagia. 1998; 13(4): 208-12.

11. de Larminat V, Montravers $\mathrm{P}$, Dureuil B, Desmonts JM. Alteration in swallowing reflex after extubation in intensive care unit patients. Crit Care Med. 1995; 23(3): 486-90.

12. Macht, M, Wimbish T, Clark BJ, Benson AB, Burnham EL, Williams A, Moss M. Diagnosis and treatment of post-extubation dysphagia: Results from a national survey. Journal of Critical Care. 2012; 27: 578-86.

13. Skoretz SA, Flowers HL, Martino R. The incidence of dysphagia following endotracheal intubation: a systematic review. Chest. 2010; 137(3): 665-73.

14. Bone DK, Davis JL, Zuidema GD, Cameron JL. Aspiration pneumonia: prevention of aspiration in patients with tracheostomies. Ann Thorac Surg. $1974 ; 18(1): 30-7$.

15. Spray SB, Zuidema GD, Cameron JL. Aspiration pneumonia: incidence of aspiration with endotracheal tubes. Am J Surg. 1976; 131(6): 701-3.

16. Elpern EH, Scott MG, Petro L, Ries MH. Pulmonary aspiration in mechanically ventilated patients with tracheostomies. Chest. 1994; 105(2): 563-6.

17. Tolep K, Getch CL, Criner GJ. Swallowing dysfunction in patients receiving prolonged mechanical ventilation. Chest. 1996; 109(1): 167-72.

18. Altman KW, Yu GP, Schaefer SD. Consequence of dysphagia in the hospitalized patient impact on prognosis and hospital resources. Arch Otolaryngol Head Neck Surg. 2010; 136: 784-9.

19. Moraes, DP, Sassi FC, Mangilli LD, Zilberstein B, Andrade CRF. Clinical prognostic indicators of dysphagia following prolonged orotracheal intubation in ICU patients. Critical Care. 2013; 17: R243.

20. Rumbach AF, Ward EC, Cornwell PL, Bassett LV, Muller JM. Clinical progression and outcome of dysphagia following thermal burn injury: a prospective cohort study. J Burn Care Res. 2012; 33: 336-46. 
21. Barker J, Martino R, Reichardt B, Hickey EJ, Ralph-Edwards A. Incidence and impact of dysphagia in patients receiving prolonged endotracheal intubation after cardiac surgery. Can J Surg. 2009; 52 (2): 119-24.

22. Ward EC, Green K, Morton AL. Patterns and predictors of swallowing resolution following adult traumatic brain injury. J Head Trauma Rehabil. 2007; 22: 184-91.

23. Medeiros GC, Sassi FC, Mangilli LD, Zilberstein B, Andrade CRF. Clinical dysphagia risk predictors after prolonged orotracheal intubation. Clinics. 2014; 69 (1): 8-14.

24. Suiter DM, Leder SB. Clinical utility of the 3-ounce water swallow test. Dysphagia. 2008; 23: 244-50.

25. Martino R, Silver F, Teasell R, Bayley M, Nicholson G, Streiner DL, Diamant NE. The Toronto bedside swallowing screening test (TOR-BSST) Development and validation of a dysphagia screening tool for patients with stroke. Stroke. 2009; 40: 555-61.

26. Edmiaston J, Connor LT, Steger-May K, Ford AL. A simple bedside stroke dysphagia screen, validated against videofluoroscopy, detects dysphagia and aspiration with high sensitivity. Journal of Stroke and Cerebrovascular diseases. 2014; 23(4): 712-16.

27. Bordon A, Bokhari R, Sperry J, Testa D, Feinstein A, Ghaemmaghami V. Swallowing dysfunction after prolonged intubation: analysis of risk factors in trauma patients. Am J Surg. 2011; 202 (6): 679-82.

28. Macht M, Wimbish T, Clark BJ, Benson AB, Burnham EL, Williams A, Moss M. Postextubation dysphagia is persistent and associated with poor outcomes in survivors of critical illness. Critical Care. 2011; 15 (R231): 1-9.

29. Macht M, King CJ, Wimbish T, Clark BJ, Benson AB, Burnham EL, Williams A, Moss M. Post-extubation dysphagia is associated with longer hospitalization in survivors of critical illness with neurologic impairment. Critical Care. 2013; 17 (R119): 1-9. 
30. Brodsky, BM, Gellar JE, Dinglas VD, Colantuoni E, Mendez-Tellez PA, Shanholtz C, Palmer JB, Needham DM. Duration of oral endotracheal intubation is associated with dysphagia symptoms in acute lung injury patients. Journal of Critical Care. 2014; 29: 574-79.

31. American Speech-Language Hearing Association National Outcome Measurement System (NOMS): Adult Speech-Language Pathology training manual. Rockville, Md: American Speech-Language Hearing Association; 1998.

32. Wesling $M$, Brady $S$, Jensen $M$, Nickell $M$, Statkus D, Escobar $N$. Dysphagia outcomes in patients with brain tumors undergoing inpatient rehabilitation. Dysphagia. 2003; 18(3):203-10.

33. Moreno R, Vincent JL, Matos R, Mendonça A, Cantraine F, Thijs L, Takala $\mathrm{J}$, Sprung $\mathrm{C}$, Antonelli M, Bruining $\mathrm{H}$, Willatts $\mathrm{S}$. The use of maximum SOFA score to quantify organ dysfunction /failure in intensive care. Results of a prospective, multicenter study. Intensive Care Med. 1999; 25: 686-96.

34. Antonelli M, Moreno R, Vincent JL, Sprung CL, Mendoça A, Passariello M, Riccioni L, Osborn J. Aplication of SOFA score to trauma patients. Intensive Care Med. 1999; 25: 389-94.

35. Solh EA, Okada M, Bhat A, Pietrantoni C. Swallowing disorders post orotracheal intubation in the elderly. Intensive Care Med. 2003; 29: 1451-55.

36. McMicken BL, Muzzy CL. Prognostic indicators of functional outcomes in first time documented acute stroke patients following standard dysphagia treatment. Disabil Rehabil. 2009; 31: 2196-2203.

37. Mackay LE, Morgan AS, Bernstein BA. Factors affecting oral feeding with severe traumatic brain injury. Head Trauma Rehabil. 1999; 14: 435-47.

38. Schoeder MF, Daniels SK, McClain M, Corey DM, Foundas AL. Clinical and cognitive predictors of swallowing recovery in stroke. J Rehabil Res Dev. 2006; 43: 301-10. 
39. Leder S, Suiter DM. An epidemiologic study on aging and dysphagia in the acute care hospitalized population: 2000-2007. Gerontology. 2009; 55: 714-18.

40. Cook IJ, Welman MD, Wallace K, Shaw DW, McKay E, Smart RC, Butler SP. Influence of ageing on oral-pharyngeal bolus transit and clearance during swallowing: scintigraphic study. Am J Physiol. 1994; 266: G972-77.

41. Yoshikawa M, Yoshida M, Nagasaki T, Tanimoto K, Tsuga K, Tsuga K, Akagawa $\mathrm{Y}$, Komatsu T. Aspects of swallowing in healthy dentate elderly persons older than 80 years. J Gerontol A Biol Med Sci. 2005; 60(4): 506-9.

42. Kullman DA, Plato CC, Tobin JD. The role of muscle loss in the age-related decline of grip strength: cross-sectional and longitudinal perspectives. $J$ Gerontol. 1990; 45M: 82-88.

43. Clarkson PM, Kroll W, Melchionda AM. Age, isometric strength, rate of tension development of fibre type composition. J Gerontol. 1981: 36: 648-53.

44. Dejaeger E, Pelemans W, Ponette E, Joosten E. Mechanisms involved in postdeglutition retention in elderly. Dysphagia. 1997; 12: 63-7.

45. Skoretz SA, Yau TM, Ivanov J, Granton JT, Martino R. Dysphagia and associated risk factors following extubation in cardiovascular surgical patients. Dysphagia. 2014; 29: 647-54.

46. Kwok AM, Davis JW, Cagle KM, Sue PL, Kaups KL. Post-extubation dysphagia in trauma patients: it's hard to swallow. The American Journal of Surgery. 2013; 206: 924-28.

47. Burgess GE, Cooper JR, Marino RJ, Peuler MJ, Warriner RA. Laryngeal competence after tracheal extubation. Anesthesiology. 1979; 51(1):73-7.

48. Stauffer JL, Olson DE, Petty TL. Complications and consequences of endotracheal intubation and tracheotomy. Am J Med. 1981; 70(1): 65-76.

49. Morinièri S, Beutter $P$, Boiron $M$. Sound component duration of healthy human pharyngoesophageal swallowing: a gender comparison study. Dysphagia. 2006; 175-82. 
50. Cichero JAY, Murdoch BE. Acoustic signature of the normal swallow: characterization by age, gender, and bolus volume. Ann Otol Rhinol Laryngol. 2002; 111(7 Pt 1): 623-32.

51.Zenner PM, Losinski DS, Millis $\mathrm{RH}$. Using cervical auscultation in the clinical dysphagia examination in long-term care. Dysphagia. 1995; 10(1): 27-31.

52. Lazareck LJ, Moussavi ZMK. Classification of normal and dysphagic swallows by acoustical means. IEEE Trans Biomed Eng. 2004; 51(12): 2103-12.

53. Mann G, Hankey GJ. Initial clinical and demographic predictors of swallowing impairment following acute stroke. Dysphagia. 2001; 16(3): 208-15.

54. Mari F, Matei M, Ceravolo MG, Pisani A, Montesi A, Provinciali L. Predictive value of clinical indices in detecting aspiration in patients with neurological disorders. J Neurol Neurosurg Psychiatry. 1997; 63(4): 456-60.

55. Kuhlemeier KV, Yates $\mathrm{P}$, Palmer JB. Intra- and interrater variation in the evaluation of videofluorographic swallowing studies. Dysphagia. 1998; 13(3): 142-7.

56. McCullough GH, Wertz RT, Rosenbek JC, Millis RH, Ebb WG, Ross KB. Inter- and intrajudge reliability for videofluoroscopic swallowing evaluation measures. Dysphagia. 2001; 16(2): 110-11. 